\title{
Increasing Silicone Oil Viscosity Increases Resistance to Volume Displacement in an in vitro Retinal Detachment Model
}

\author{
Alexander Rubowitz $^{a} \quad$ Leena Asalee $^{a} \quad$ Uri Zaretski $^{b}$ \\ aDepartment of Ophthalmology, Meir Medical Center, attributed to Sackler School of \\ Medicine, Tel Aviv University, Kfar Saba, Israel; b Department of Biomedical Engineering, \\ Tel Aviv University, Tel Aviv, Israel
}

\section{What Is It about?}

- Retinal detachment is an important blinding morbidity and has several risk factors (myopia, trauma, specific syndromes, etc.). Currently, one of the most common repair techniques for retinal detachment combines pars plana vitrectomy, laser, or cryotherapy and placement of a vitreous substitute into the vitreous cavity such as silicone oil. We examined whether higherviscosity silicone oils offer greater resistance to volume displacement in an in vitro eye model of retinal detachment, and could subsequently be expected to generate stronger retinal tamponades in cases of recurrent or complex retinal detachments.

\section{Keywords}

Silicone oil viscosity $\cdot$ Volume displacement $\cdot$ Retinal detachment

\begin{abstract}
Purpose: To determine whether higher-viscosity silicone oils offer greater resistance to volume displacement in an in vitro eye model of retinal detachment and could subsequently be expected to generate stronger retinal tamponades in cases of recurrent or complex retinal detachments. Methods: We used an eye model that included a membrane representing the retina. Silicone oils with different viscosities were injected, and oil displacement and resistance were measured. Results: Membrane movement and volume changes in an eye model were proportional to the viscosity of the oil: the lower the viscosity, the faster volume changes and displacement occurred. Conclusions: Our results show that the volume change rate has a linear relationship with oil viscosity. Our findings suggest that in this eye model, silicone oil tamponades the retina against the retinal pigment epithelium mainly through volume displacement and that silicone oil viscosity may play an important role in preventing retinal displacement and in providing tamponade.




\section{Introduction}

Retinal detachment is a significant cause of morbidity. Currently, one of the most common repair techniques for retinal detachment combines pars plana vitrectomy, laser, or cryotherapy and placement of a vitreous substitute into the vitreous cavity [1].

Although vitreous substitutes have gradually improved, the field is poised to introduce novel compounds that result in improved surgical outcomes [1]. Silicone oils (polydimethylsiloxanes) have been used since 1962 for complex retinal detachments, for inferior or giant tears, or in patients unable to position themselves after surgery with gas [1]. Complications include emulsification, subretinal silicone oil migration, tissue toxicity, cataract, glaucoma, and keratopathy, among others [1].

To be effective as an internal tamponade, silicone oil must displace aqueous fluid (which replaces vitreous fluid after surgery) away from the retinal surface. Several studies have investigated the mechanism of action of silicone oil tamponade [1-3]. Based on published articles and textbooks, four physical parameters influencing this function have been widely accepted. The first is specific gravity, which explains why an intraocular tamponade agent sinks or floats in aqueous humor. As the specific gravity of silicone oil is slightly lower than that of vitreous ( 0.97 vs. 1.00 ), it floats in the vitreous cavity [3]. The second parameter is buoyancy. An intraocular bubble of tamponade material is acted upon by two opposing forces: buoyancy (upward force) and gravity (downward force). Regarding the vitreous cavity, the result is the force with which the bubble presses against the superior retina. For silicone oil, this force is relatively small, as the specific gravity is very close to that of aqueous or vitreous fluid [3]. The third parameter is interfacial tension. When two immiscible agents are used together (e.g., silicone oil and aqueous fluid), the surface tension between them is called interfacial tension. Gas or air has the highest interfacial tension against water (approx. $80 \mathrm{mN} / \mathrm{m}$ ), whereas perfluorocarbon liquids and silicone oils have lower interfacial tensions of 40-45 and $35 \mathrm{mN} / \mathrm{m}$, respectively [3]. The last parameter is viscosity, which is the physical property of a fluid's resistance to gradual deformation by shear stress. The tendency of a substance to emulsify and to disperse into droplets over time is also dependent on its viscosity.

Physical problems with each of these theories make the exact mechanism by which silicone oil exerts tamponade on the retina uncertain. Water has the highest surface tension of any fluid (except liquid mercury): approximately $73 \mathrm{mN} / \mathrm{m}$, which is twice that of silicone oil. However, water in the vitreous cavity does not prevent retinal detachment and does not provide effective retinal tamponade. Therefore, surface tension of the vitreous cavity fluid against the retina may not be the main mechanism of tamponade.

Previous trials using viscoelastic materials [4-9], mainly hyaluronic acids, as tamponade agents in retinal detachment surgeries found that they performed well for short-term tamponade. However, retinal detachments recurred frequently due to the short half-life of these materials in the eye. They have the same specific gravity, buoyancy, and surface tension as water, and would not be expected to tamponade any better than water does for retinal detachment. Therefore, it appears that these may also not be the major mechanisms of silicone oil action on retinal detachment.

Volume displacement, which we hypothesize to be the main mechanism of retinal tamponade, acts by mechanically displacing preretinal and subretinal fluid away from the site of retinal breaks and apposing the retina to the underlying retinal pigment epithelium [8] by exerting a volume-filling force in the vitreous. This mechanism is often used in anterior segment surgeries to fill and stabilize the anterior chamber during lens manipulations. Higher-viscosity materials, such as Healon5 (Abbott Medical Optics, Abbott Laboratories Inc., Abbott Park, IL, USA) or Biolon Prime (Bio-Technology General Ltd., Kiryat Malachi, Israel), cause a stronger filling effect by pushing aside and expanding the anterior chamber to a 


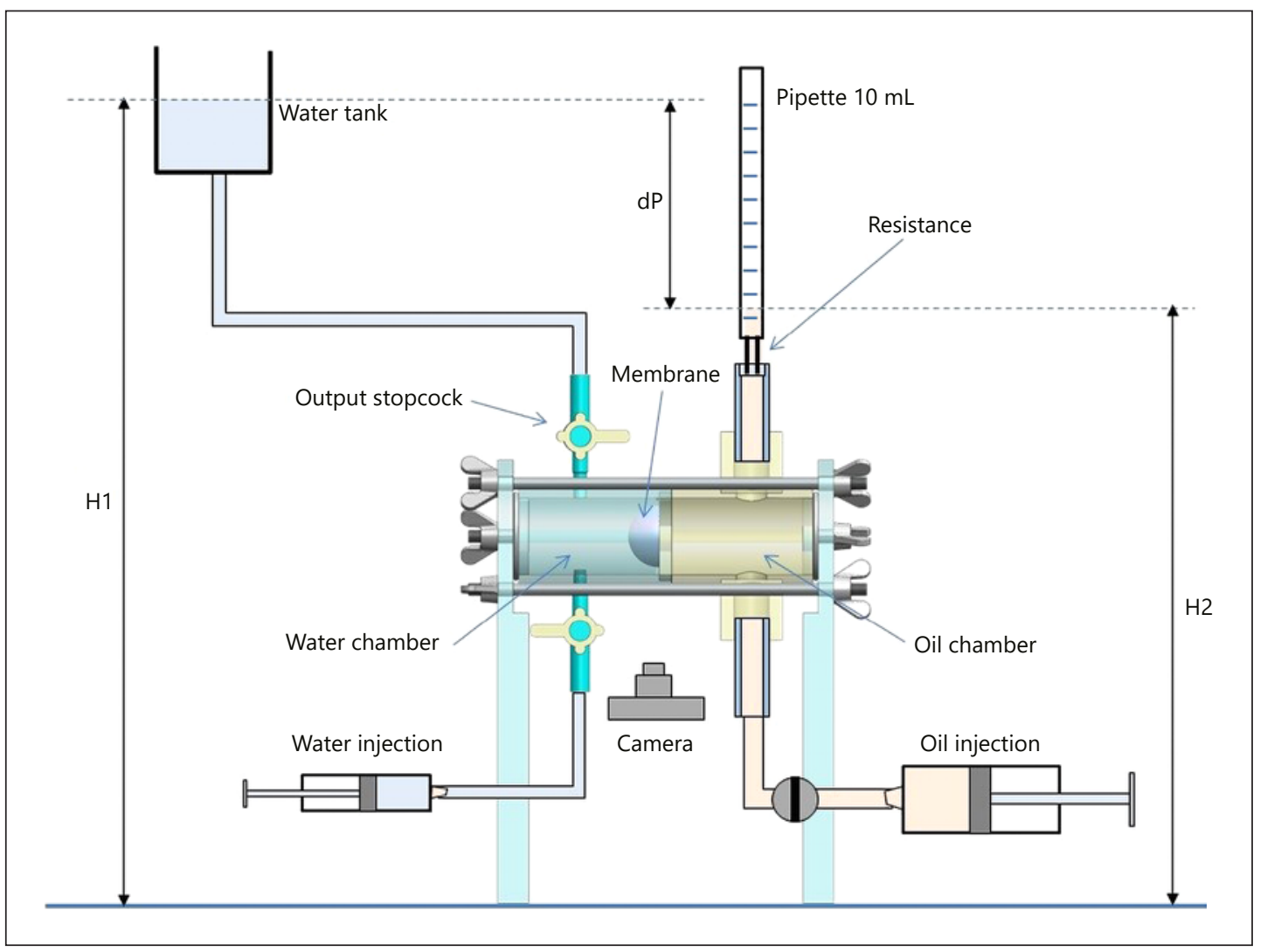

Fig. 1. Eye model schematic diagram.

greater degree than less viscous materials (e.g., Healon or Biolon) do. This is easily seen during anterior segment surgery.

The goal of this study was to elucidate the mechanism by which silicone oil tamponades retinal detachment and to determine whether higher-viscosity silicone oils may generate stronger retinal tamponade.

\section{Methods}

The study was performed at the Department of Biomedical Engineering at Tel Aviv University. An eye model employing a membrane representing the retina was used. The displacement and resistance of fluid for various viscosity silicone oils were measured.

\section{Simulation Setup}

The design of the eye model and simulation setup are shown in Figure 1. It consists of two Perspex transparent cylinders (25-mm internal diameter). The cylinders are separated by a hemispheric convex membrane simulating the retina, made of $0.1-\mathrm{mm}$ thick latex with a 10 -mm radius of curvature.

The chamber on the convex side of the membrane was filled with water and the chamber on the concave side was filled with silicone oil. The volume of each chamber was $30 \mathrm{~mL}$ and the two chambers were completely separated by the membrane, with no contact between them. 
Fig. 2. Eye model.

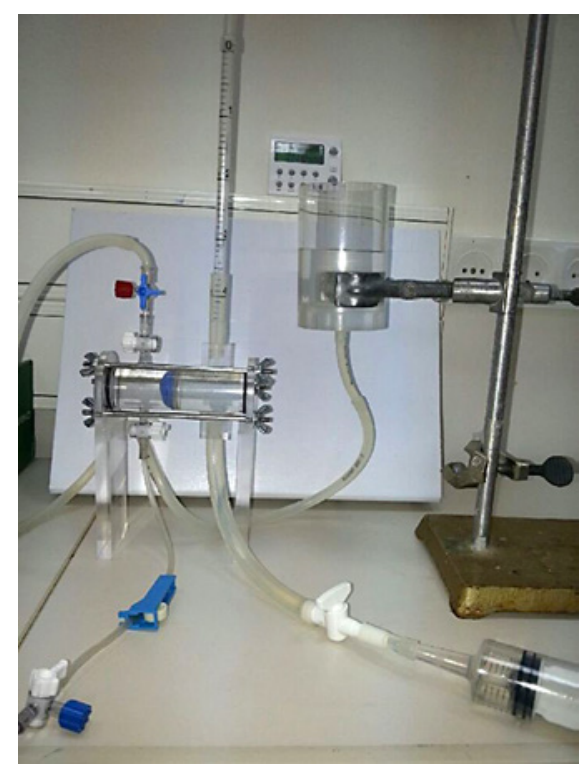

Water and oil were inserted through injection stopcocks at the bottom of each chamber, to eliminate air bubble formation in the chambers.

The pipette was marked in 0.01-mL intervals.

Working with the Setup

The left water chamber was filled to where the water level was equal to the oil level in the right chamber pipette. The shape of the membrane and the fluid levels were allowed to stabilize for at least 5 min (Fig. 1, 2).

The water and oil injection stopcocks below the chambers were closed during the test and measurement, and were used only to fill the chamber prior to each experiment.

We then closed the water chamber output stopcock and raised the water tank to establish a prespecified water pressure gradient. Next, we opened the stopcock and observed the oil level rising in the silicone oil chamber pipette due to the pressure gradient from the raised water tank. At the same time, we measured the volume changes in the oil pipette in 10-s intervals and observed the changes in the contour of the latex membrane. The experiment was stopped when the membrane became convex (Fig. 3a-e).

To return to the initial state, we lowered the water tank to its initial height, again waiting for stabilization. At least three test measurements were performed for each of the four silicone oil viscosities used.

\section{Data Analysis}

The average values of volume change were calculated. The average volume changes over time were plotted for each oil viscosity tested. The dependencies were interpolated by straight lines with correlation coefficient $\mathrm{R}^{2}$. The time needed for a 1-mL volume change was another parameter that was calculated, which is more convenient for medical purposes (and more readily intuitive to physicians).

\section{Results}

Figures 4 shows the change in volume of silicone oil over time, in minutes. The higher the viscosity, the slower the change in volume over time. 


\section{Biomedicine क्रमापा}

Fig. 3. Changes in membrane shape due to fluid volume changes.

Fig. 4. Change in volume of silicone oil over time according to viscosity.
C) 2018 The Author(s). Published by S. Karger AG, Basel www.karger.com/bmh

Rubowitz et al.: Increasing Silicone Oil Viscosity Increases Resistance to Volume Displacement in an In Vitro Retinal Detachment Model
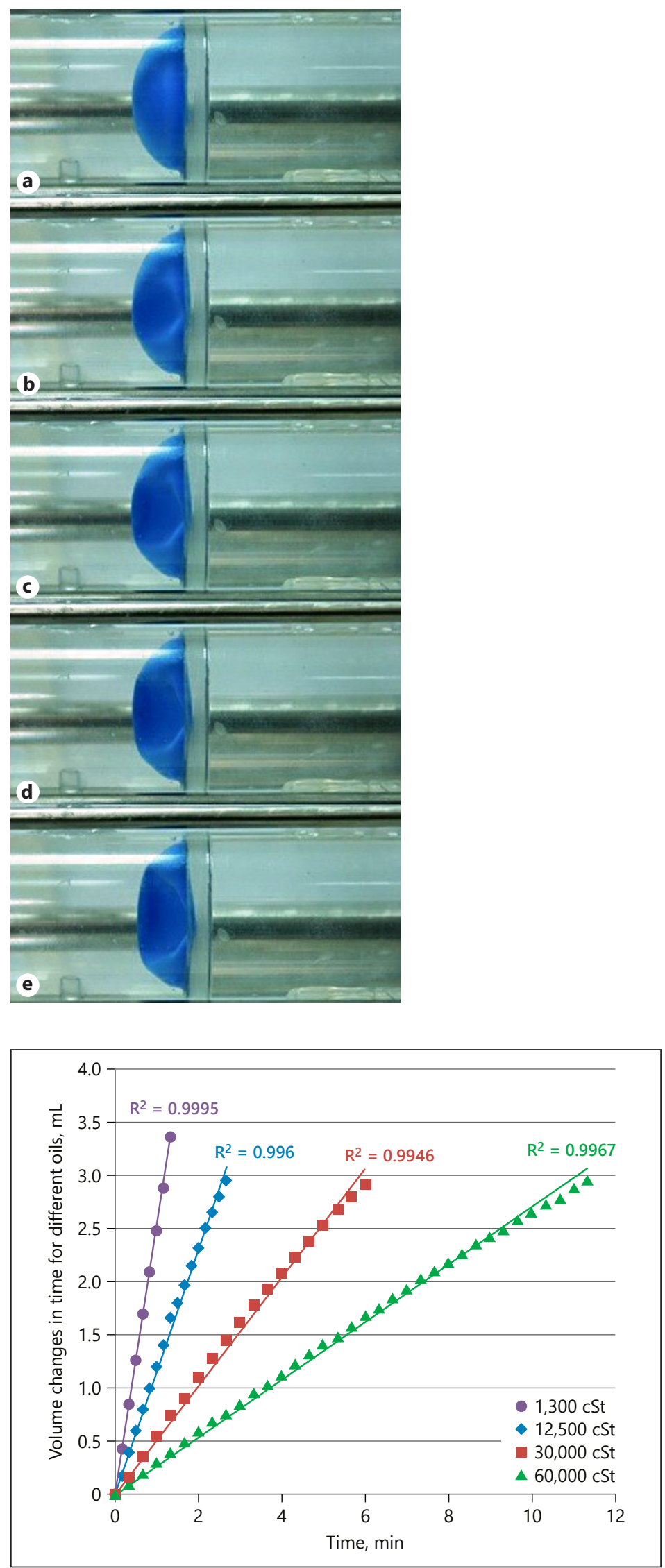
Fig. 5. Time (in s) for a 1-mL volume change for the different viscosity oils.

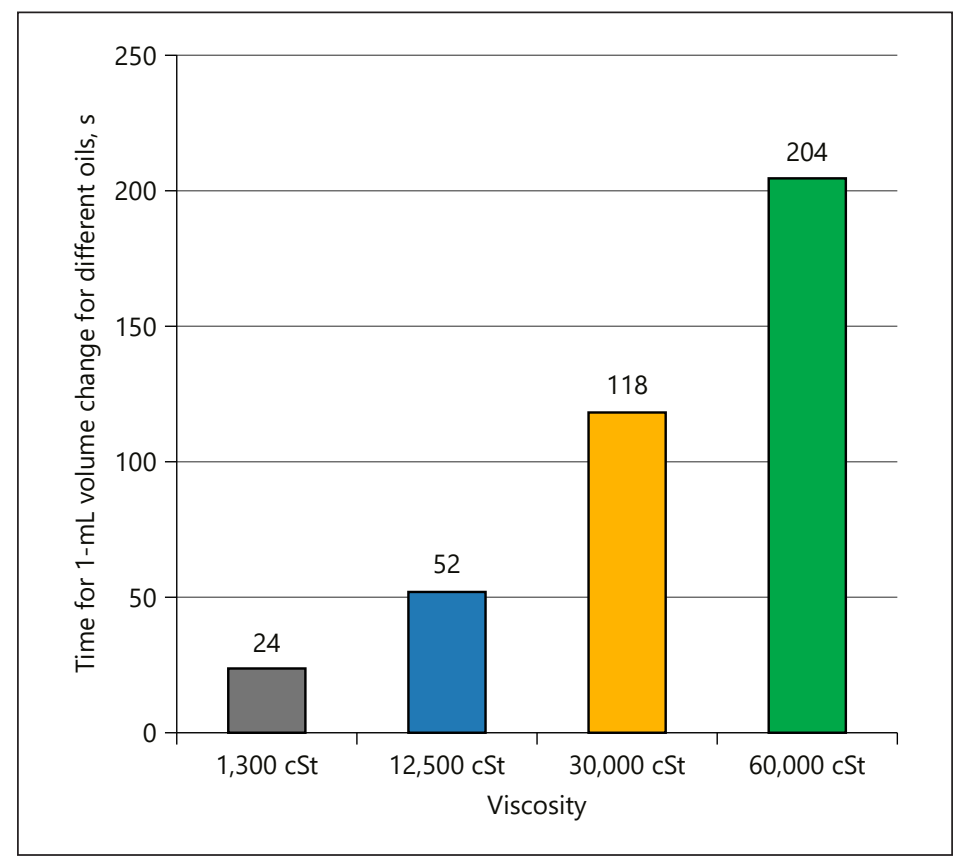

Figure 5 shows the time for a $1-\mathrm{mL}$ volume change for the different oils. The silicone oil with the highest viscosity displayed the slowest change in volume over time.

\section{Discussion}

As described in the introduction, four mechanisms have been hypothesized to explain the tamponade function of silicone oil in retinal detachment: surface tension, specific gravity, buoyancy, and volume displacement.

In an attempt to show that volume displacement is the primary mechanism by which silicone oils exert their tamponade action on a detached retina, we built a simulator in which buoyancy, surface tension, and specific gravity were not relevant factors. We did this by eliminating any contact between silicone oil and any other fluid including water, so that the silicone oil did not float or exert surface tension against any liquid. Flow resistance and volume change and displacement were the only mechanisms acting in the system, as represented in Figures 3-5.

If surface tension, specific gravity, and buoyancy were indeed the major effectors of tamponade in retinal detachment, as previous literature has suggested, there would seem to be no point in investigating the effectiveness of higher-viscosity oils for failed or severe detachments, as all silicone oils have the same specific gravity, surface tension, and buoyancy irrespective of their viscosity. However, if the primary tamponading mechanism were volume displacement, then higher-viscosity oils would be expected to resist volume displacement more efficiently and might be more effective than the silicone oil viscosities currently used for recurrent detachments.

Using this in vitro model of retinal detachment, we sought to elucidate the mechanism by which silicone oil exerts its tamponading effect in treating retinal detachment. By preventing contact between the silicone oil and water, the model would seem to eliminate the proposed mechanisms of surface tension and buoyancy, and to measure the effect of volume displace- 
ment exclusively. The results suggest that silicone oil tamponade in retinal detachment may act via a volume displacement mechanism in the vitreous cavity, and that viscosity may therefore play an important role in its resistance to volume displacement.

There are several limitations to this study. The use of an in vitro model comprised of a plastic outer shell, with a membrane simulating the retina, may not take into account varying and individual characteristics of these tissues in the human eye. The behavior of silicone oil in an eye with retinal detachment may be influenced by numerous physical qualities of these tissues which a model cannot simulate, including the precise flexibility and porosity of each tissue, the stretching and elastic abilities of the various eye structures, intraocular temperature, and the subjection of the eye to continuous ocular and head movements, to name a few. In addition, the model did not allow contact between the silicone oils and water (which exists in the eye in vivo) in order to differentiate the effects of volume displacement from the effects of buoyancy, surface tension, and liquid interfaces. We used this method to show that volume displacement, both theoretically and in this model, is an important, if not major effector of the clinical tamponade effect of the oil. In the eye, however, there are oil-water interfaces, oilretina interfaces, and oil-lens interfaces (phakic or pseudophakic), all influencing the tamponade effect of the oil in the eye.

\section{Conclusion}

The results presented here suggest that volume displacement, rather than the conventionally described mechanisms of surface tension and buoyancy, may be a major effector of silicone oil tamponade in retinal detachment. There are strong theoretical reasons to support this proposal. In this study using a model for retinal detachment, we believe we have shown some evidence that volume displacement is a major mechanism of tamponade. As we have shown, the viscosity of the silicone oil has a major role in influencing volume change over time.

Were surface tension and buoyancy the main tamponade mechanisms of silicone oils in the eye, there would be no point in studying higher viscosity oils, as they all share the same surface tension and specific weight characteristics. However, if volume displacement is a major tamponade mechanism, higher viscosity oils may exert stronger volume filling and tamponading effects on the retina.

In light of this evidence in an in vitro model of retinal detachment, we believe that higherviscosity silicone oils may provide superior tamponade, especially in cases of complex or recurrent detachments, and that higher-viscosity silicone oils should be studied further for efficacy in animal models and in a clinical setting in real eyes.

\section{Disclosure Statement}

The authors have no proprietary, funding, or conflicts of interest to disclose. The study had no financial support. 
Rubowitz et al.: Increasing Silicone Oil Viscosity Increases Resistance to Volume

Displacement in an In Vitro Retinal Detachment Model

\section{References}

1 Foster WJ. Vitreous Substitutes. Expert Rev Ophthalmol. 2008 Apr;3(2):211-8.

2 Petersen J. The physical and surgical aspects of silicone oil in the vitreous cavity. Graefes Arch Clin Exp Ophthalmol. 1987;225(6):452-6.

3 Barca F, Caporossi T, Rizzo S. Silicone oil: different physical proprieties and clinical applications. Biomed Res Int. 2014;2014(June):502143.

4 Folk JC, Packer AJ, Weingeist TA, Howcroft MJ. Sodium hyaluronate (Healon) in closed vitrectomy. Ophthalmic Surg. 1986 May;17(5):299-306.

5 Vatne HO, Syrdalen P. The use of sodium hyaluronate (Healon) in the treatment of complicated cases of retinal detachment. Acta Ophthalmol (Copenh). 1986 Apr;64(2):169-72.

6 Gerke E, Meyer-Schwickerath G, Wessing A. Healon in retinal detachment with proliferative vitreoretinopathy. Graefes Arch Clin Exp Ophthalmol. 1984;221(5):241-3.

7 Pruett RC, Schepens CL, Swann DA. Hyaluronic acid vitreous substitute. A six-year clinical evaluation. Arch Ophthalmol. 1979 Dec;97(12):2325-30.

8 de Juan E Jr, McCuen B, Tiedeman J. Intraocular tamponade and surface tension. Surv Ophthalmol. 1985 Jul-Aug;30(1):47-51.

9 Kanski JJ. Intravitreal hyaluronic acid injection. A long-term clinical evaluation. Br J Ophthalmol. 1975 May; $59(5): 255-6$. 